\title{
Integrating a 19F MRI Tracer Agent into the Clinical Scale Manufacturing of a T-Cell Immunotherapy
}

\author{
Charles F. O'Hanlon, ${ }^{1}$ Tamara Fedczyna, ${ }^{2}$ Shannon Eaker, \\ William D. Shingleton, ${ }^{3}$ and Brooke M. Helfer ${ }^{1}$ \\ ${ }^{1}$ Celsense Inc., 603 Stanwix Street Suite 390, Pittsburgh, PA 15222, USA \\ ${ }^{2}$ GE Healthcare, 100 Results Way, Marlborough, MA 01752, USA \\ ${ }^{3}$ GE Healthcare, The Grove Center, White Lion Road, Amersham, Buckinghamshire HP7 9LL, UK \\ Correspondence should be addressed to Brooke M. Helfer; brooke@celsense.com
}

Received 10 July 2017; Revised 19 September 2017; Accepted 2 October 2017; Published 2 November 2017

Academic Editor: Anne Roivainen

Copyright (C) 2017 Charles F. O'Hanlon et al. This is an open access article distributed under the Creative Commons Attribution License, which permits unrestricted use, distribution, and reproduction in any medium, provided the original work is properly cited.

\begin{abstract}
Leukocyte immunotherapies have made great progress in the treatment of cancer. Recent reports on the treatment of B-cell malignancies using Chimeric Antigen Receptor and affinity enhanced T-Cell Receptor therapies have demonstrated encouraging clinical results. As investigators begin to explore the treatment of solid tumors with these cells, the hurdle of evaluating T-cell homing to and persistence at the site of disease remain. Significant challenges regarding the GMP manufacture and administration of a therapeutic dose of millions to billions of transduced T-cells remain. Here we report on the application of a clinically authorized 19F MRI tracer agent to human T-cells, employing state-of-the-art methods and equipment in the manufacture of a cellular therapy. Using a general T-cell expansion protocol and clinical scale industrial bioreactors, we show 19F labeling without detriment to the product $+/-$ cryopreservation. While the incorporation of the $19 \mathrm{~F}$ tracer is not trivial, it is just one of the many steps that can aid in progression of a therapeutic to and though the clinic. Combining the MRI tracking capabilities, safety profiles, and clinical sensitivity of this method, this application demonstrates the ability of 19F MRI to be used in industrial scale applications to visualize the spatial fate of cellular therapeutics.
\end{abstract}

\section{Introduction}

In the past 5 years, the successful treatment of cancer patients with autologous T-cells that have been engineered to recognize and kill tumors has been reported in the academic literature [1-8]. Although the total number of patients treated is relatively small, the unexpectedly high rates of complete response have provoked global reaction by other academic investigators, large pharmaceutical companies, and financial markets to allocate significant resources to translate these therapies into commercial products that can be economically delivered to patients.

The enabling breakthrough for these new therapies is the targeting of tumor cells with aberrant patterns of gene expression versus the originating tissue type [9]. These therapies are typically referred to as CAR T-cells (Chimeric Antigen Receptor-T-cells) or TCR cells (T-Cell Receptor cells) but may involve NK cells, mixed lymphocytes, and T-cells and NK cells derived from allogenic cell lines. In this study, we refer to them generically as engineered T-cell therapies. The engineered aspect of these cells typically involves genetically modifying immune cells in culture to express a receptor with an affinity for a target expressed by the tumor cells [10].

A second engineering aspect is large scale processes to genetically modify tens of millions of cells in culture, extracting the "unused" proteins and virus used in the transduction process, expanding the population of modified cells, and testing the cell product to verify that it meets predetermined release criteria. Integrating manufacturing of complex proteins and virus particles, traditional tissue culture, large scale transduction, and quality assurance on an industrial scale in a patient-centric process is a daunting undertaking that is currently being developed in real-time on a global basis $[10,11]$. This process involves many empirical 
steps: testing a particular technology on a stand-alone basis and retesting the technology integrated in the putative large scale process.

The rich market valuations for companies specializing in engineered T-cell therapies indicate that investors expect therapeutic developers to rapidly overcome the many manufacturing challenges for these treatments and advance beyond the treatment of blood cancers to solid tumors. Of the 1.7 million new cases of cancer in the US each year, less than $1 \%$ involve Acute Lymphoblastic Leukemia (ALL), the most successful treatment to date, and less than $10 \%$ involve leukemia, lymphoma, and myeloma [12].

Investigators and developers of engineered T-cell therapies are currently considering a large number of receptors and targets to treat solid tumors. Unfortunately, there is no high throughput in vitro method or data demonstrating the relevancy of preclinical models involving xenograft human tumors and engineered human immune cells. Like the work in ALL, the path forward will involve multiple early clinical studies. To accelerate the process, developers must embrace new technologies that answer fundamental questions regarding migration, persistence, and tumor killing for new receptor and target combinations. Definitive pharmacokinetic (PK) data for engineered T-cells will rapidly identify promising therapies and may provide further guidance for dosing strategies, different routes of administration, the ablation of endogenous immune cells, and the simultaneous administration of check point inhibitors or other immunotherapies.

The ideal cellular imaging technology for the development of PK data meets several criteria including being a quantitative, sensitive, biocompatible (viability preserving and nongenetically modifying), long lived, and nondilutive [13]. Most imaging technologies meet some but not all of these characteristics, with varying pros and cons of each modality, requiring a balance of wants and needs in examining cellular migration and persistence. Common imaging modalities for clinical cell tracking include MRI, PET, and SPECT. Ultrasound has also been used but is less common (imaging modalities reviewed extensively elsewhere, [1317]). Here we will examine a nonradioactive perfluorocarbon based imaging agent that has been used clinically and is able to be visualized and quantified by fluorine MRI $[18,19]$.

Incorporation of fluorine labeling has been well demonstrated in the literature [18-24] but only on a small scale, cultures involving small volume flasks and not liters of media. In this paper we describe one of the empirical steps in the process of evaluating a new therapeutic component, namely, cell labeling. Here we report on a set of experiments that demonstrate that Cell Sense (CS-1000), a 19F MRI cell tracking agent used to develop clinical pharmacokinetic data for cell therapies, can be integrated into an industrial process for manufacturing an engineered T-cell therapy.

\section{Methods}

2.1. T-Cell Generation. Lymphocytes were isolated from buffy coats (Central Blood Bank, Pittsburgh PA) by Ficoll (GE Healthcare) and gradient separation (Percoll, GE Healthcare) as described previously [25]. Lymphocytes were stimulated with $2.5 \mathrm{ug} / \mathrm{mL}$ PHA (Roche) and cultured in Iscove's Modified Dulbecco's Medium (IMDM, Gibco) containing 10\% human AB serum (Sigma), 200 IU/mL IL-2 (GE Healthcare), 0.5\% Penicillin, Streptomycin, Glutamine mix (PSG, Gibco), and $600 \mathrm{mg} / \mathrm{L}$ glucose (Gibco).

2.2. Cellular Expansion. Cells were expanded in 2 L Perfusion Cellbags in Wave 2/10 (Xuri W5) bioreactors with perfusion capability (GE Healthcare). Cells were initially seeded at $2.5-5 \times 10^{5}$ cells $/ \mathrm{mL}$ with $5 \% \mathrm{CO} 2$ and 6 RPM (rock per minute). Culture volume was increased, keeping cells at $5 \times$ $10^{5}$ cells $/ \mathrm{mL}$ until $1 \mathrm{~L}$ of media is reached. Once the culture volume was above $750 \mathrm{~mL}$, rock was increased to $8 \mathrm{RPM}$. Perfusion was started once culture volume reaches $1 \mathrm{~L}$ and the rock rate was increased to 10 RPM. Days 1-2 were set for $250 \mathrm{~mL}$ of perfusion. Days 3-5 were set at $500 \mathrm{~mL}$. Cultures were grown for 7 days reaching up to $2 \times 10^{6}$ cells $/ \mathrm{mL}$.

2.3. $19 F$ Labeling of Human T-Cells. Cell Sense (CS-1000 ATM, Celsense Inc.) was infused via a vented vial spike (ICU Medical) into the bioreactor. Reagent was either pumped in via the bioreactor pump or fed by gravity. Vials are filled with $27 \mathrm{~mL}$ of reagent, with the spike $25 \mathrm{~mL}$ being delivered per vial. Cells were labeled at $10 \mathrm{mg} / \mathrm{mL}$ CS-1000 ATM for the final 24 hours of culture.

2.4. Cellular Viability. Cellular viability was determined by Trypan Blue exclusion.

2.5. Cellular Phenotype. Cell surface immunostaining analysis was performed using a BD FACS Caliber flow cytometer. Fluorescein isothiocyanate (FITC) CD3 and CD4 (Beckman Coulter Immunotech) and phycoerythrin (PE) CD8 (Beckman Coulter Immunotech) and CD32 (Biolegend) conjugated antibodies were used with their associated control antibodies (Beckman Coulter Immunotech and Biolegend) to assess the cellular population. Before staining, cells were washed in phosphate buffered saline (PBS) and blocked in $20 \%$ human AB serum for 10 minutes. Samples were then incubated with fluorescent antibodies at suggested concentrations for 20 minutes. After washing with PBS, samples were analyzed. Data analysis was performed using BD CellQuest Pro Software.

2.6. Nuclear Magnetic Resonance (NMR) Analysis of Labeled Cells. To assay the fluorine content of the cells after labeling, cell pellets of a known number of cells $\left(\geq 3 \times 10^{6}\right.$ cells) were lysed with 1\% Triton-X 100 (Sigma Aldrich) and a fluorine reference solution (trifluoroacetic acid, TFA) for a final concentration of $0.05 \%$ TFA. The lysed solution was placed in a $5 \mathrm{~mm}$ quartz NMR tube and read using a Bruker AVANCE spectrometer (Bruker, Billerica MA) operating at $282 \mathrm{MHz}$. 19F NMR spectra with both PFPE and TFA peaks were obtained and the ratios of the integrated areas under the peaks were used to calculate the mean $19 F /$ cell or $F / c$ as described previously $[24,26]$. 


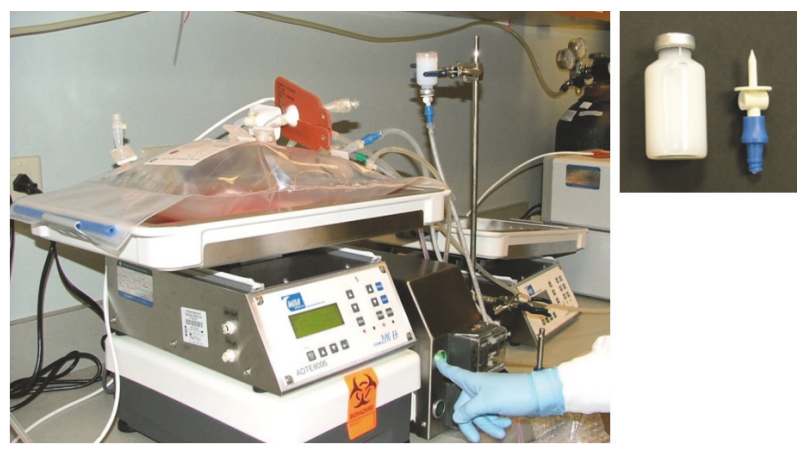

(a)

(b)

FIGURE 1: Incorporation of cellular labeling. Human T-cells were expanded to $1 \mathrm{~L}$ of culture in a GE Wave 2/10 bioreactor. (a) By adding CS-1000 ATM through a vented spike, a closed sterile system was able to be maintained. Vials containing $27 \mathrm{~mL}$ of CS-1000 ATM were fed either by gravity (not shown) or by peristaltic pump through a vented vial spike. With this method of delivery, $25 \mathrm{~mL}$ of reagent is delivered per vial. (b) $27 \mathrm{~mL}$ vial imaged with the vented spike.

2.7. Determination of Labeled Population. Cells were labeled with Cell Sense CS-ATM DM Green (CS-DM), a preclinical grade, fluorescent green conjugated version of CS-1000 ATM as performed above. Cells were washed of excess reagent and label uptake assessed by flow cytometry. Percentage of labeled population was determined by comparing the histograms for the 488 emission channel between unlabeled and CS-DM labeled cells.

2.8. Cryopreservation. Cells were pelleted and resuspended in $20 \%$ serum (Sigma Aldrich) $10 \%$ dimethyl sulfoxide (DMSO, brand) containing media which is slowly brought down to $-80^{\circ} \mathrm{C}$ before transferring to liquid nitrogen.

\section{Results}

3.1. The Logistics of Labeling at Industrial Scale. Introducing an imaging agent into clinical preparations requires the properties of the therapeutic and the manufacturing protocol for cellular preparation be unaltered by its addition. Preclinical studies have shown the efficacy of the reagent at experimental levels and in the clinic at cellular doses of $10^{6}$ cells $[18,20-$ $24]$. In the area of T-cell therapies, higher doses of cells and large volumes are often required for cellular expansion. Scale, in this context, takes into consideration billions of cells and its relationship to the volume of label, along with the ability to maintain sterility within the system. These parameters at a small scale were not an obstacle when compared to larger clinical scale preparations. The issue of scale was addressed by taking product packaging volume from $4 \mathrm{~mL}$ to $27 \mathrm{~mL}$. The $27 \mathrm{~mL}$ packaging resulted in $25 \mathrm{~mL}$ of delivered reagent with $2 \mathrm{~mL}$ of hold-up volume when administered by vented spike (Figure 1). Small scale preparations involved the addition of reagent to a flask in a tissue culture hood, while large scale preparations are often performed in a clean room, where a closed system is preferred. The addition of the larger packaging size and vented spike delivery allows for larger volume administration without the transfer of the bioreactor to a culture hood. Using this method, reagent can be pumped in via the bioreactors peristaltic pump or simply gravity fed. Adding to the adaptability of the process, cellular labeling was performed in the final 24 hours of cellular expansion as not to increase the time or complexity of product preparation.

3.2. Uptake and Viability. Cellular viability is a common release criterion for therapeutic cells. Keeping the requirements for an imaging reagent in mind, cells were examined under two categories: normal healthy donor expanded T-cells and cryopreserved T-cells. The two categories of cells were used to examine clinical scale labeling and expansion as well as the storage of labeled cells to assess labeling properties for stored and/or shipped cells. Prior studies showed that Tcells are labeled effectively at $10 \mathrm{mg} / \mathrm{mL}$, which is the constant dose used throughout the studies (data not shown). Cells were labeled at $10 \mathrm{mg} / \mathrm{mL}$ in $1 \mathrm{~L}$ of media for 24 hours. The label possesses a single major spectral peak by NMR, which when compared to a trifluoroacetic acid standard (TFA) allows for the calculation of $F / c(19 F /$ cell, Figure 2(a)). All cell groups demonstrated labeling of $10^{11} 19 F /$ cell $(F / c)$. Using Trypan Blue exclusion, we clearly demonstrated that cellular viability was maintained (Figures 2(c) and 2(d)). Importantly, cryopreserved cells maintain cellular health and retain label after thaw (Figure 2(c)). Viability and $F / c$ are summarized for each category of cells in Figure 2(d). Examining the degree of label retention in nonengineered cells, a fluorescently conjugated form of Cell Sense was used to show the distribution of cellular label (Figure 2(b)). Flow cytometry confirmed that $\sim 90 \%$ of the T-cell preparation was labeled with reagent.

3.3. Cellular Characterization. A cellular therapeutic requires a clear definition and characterization of the cellular product. Before therapeutics is released for patient administration, a number of release criteria are assessed. A panel of surface markers was used to assess labeled and unlabeled cells in each of the previously mentioned categories (recently expanded and cryopreserved). Different immunotherapeutic T-cells have different release criteria; in this study we examined the cellular populations for the CD3 T-cell maker, CD8 cytotoxic T-cell marker, CD4 T helper cell marker, and CD32 myeloid and B-cell maker. The ratios of CD8 and CD4 cells were maintained with a limited number of CD32 (nonlymphocyte) cells present (Figure 3). Importantly, these ratios were maintained after cryopreservation. The composition of engineered cells will vary by the way in which they are generated, with the desired ratios of CD 4 : CD8 cell varying by manufacture; based on the labeling performed here, we would not anticipate the ratios to change in other expansion protocols.

\section{Discussion}

Early Phase 1 trials primarily look for evidence of drug safety, but pharmacologic activity and early indications of efficacy are often explored. Early Phase 1 trials typically involve escalating dosage and a single route of administration. 


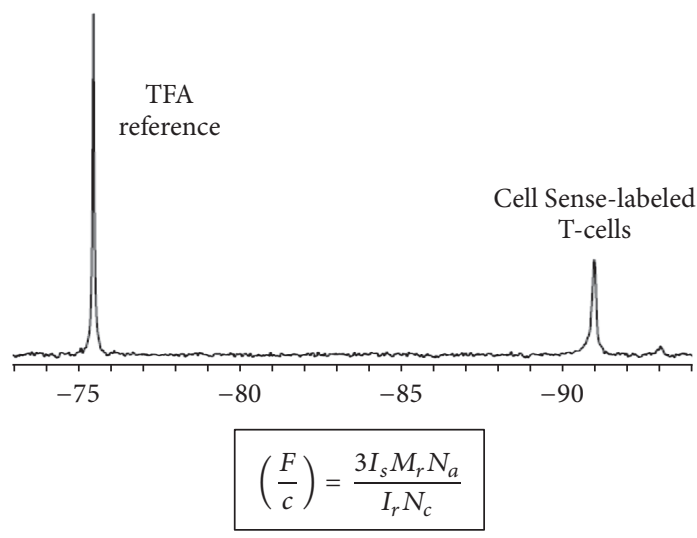

(a)

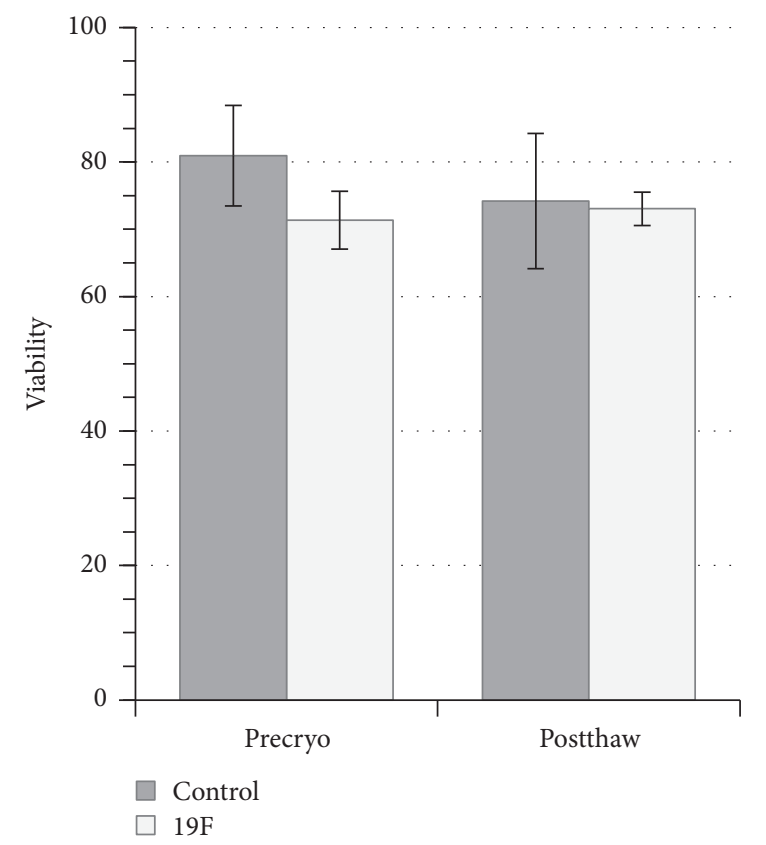

(c)
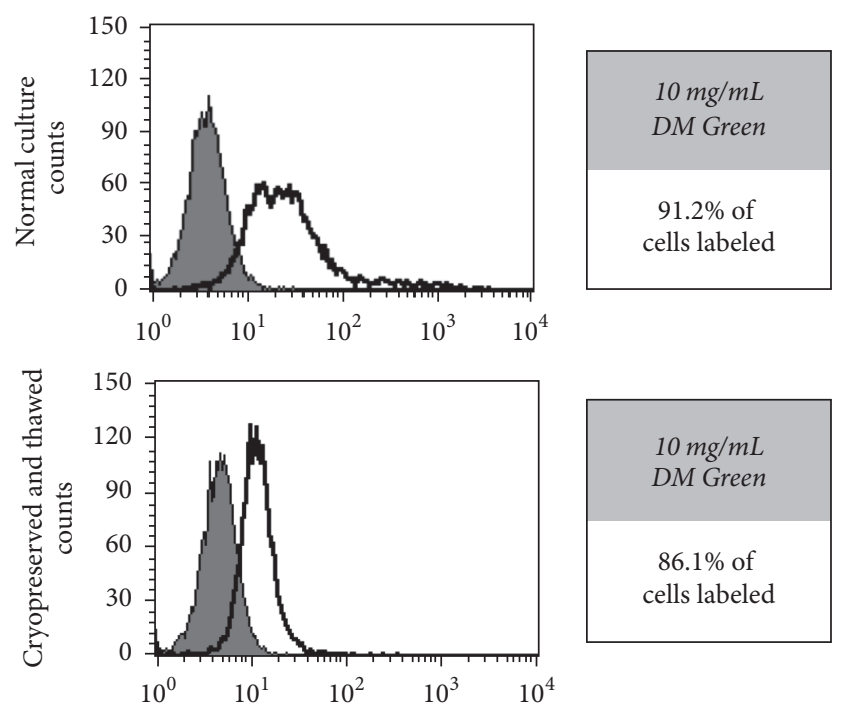

(b)

\begin{tabular}{lccc}
\hline $\begin{array}{l}\text { CS-1000 } \\
\text { concentration } \\
(\mathrm{mg} / \mathrm{mL})\end{array}$ & Population & $\begin{array}{c}\text { Viability } \\
\text { (\% of control) }\end{array}$ & $\begin{array}{c}F / c \\
\text { (average) }\end{array}$ \\
\hline 10.0 & Normal culture & $93.4 \pm 10.4$ & $\begin{array}{c}4.76 \times 10^{11} \\
\left( \pm 2.4 \times 10^{11}\right)\end{array}$ \\
10.0 & $\begin{array}{c}\text { Cryopreserved } \\
\text { and thawed }\end{array}$ & $99.8 \pm 13.6$ & $\begin{array}{c}3.43 \times 10^{11} \\
\left( \pm 4.1 \times 10^{11}\right)\end{array}$ \\
\hline
\end{tabular}

FIGURE 2: Label uptake and cellular viability after label. (a) CS-1000 ATM produces a single major spectral peak when detected by NMR. Using the integration values of the labeled cells and TFA reference, the $19 \mathrm{~F}$ atoms/cell $(F / c)$ can be calculated. Is $=$ integrated area of major peak of the cell pellet, $\mathrm{Mr}=$ moles of TFA reference (three 19F per TFA molecule already reflected in equation), Na = Avogadro's number, Ir = integrated area under TFA reference peak, and $\mathrm{Nc}=$ number of cells in pellet. (b) Labeling with a green fluorescently conjugated version of the reagent, cellular uptake of the reagent was examined by flow cytometry (n2). (c) Comparison of the viability of labeled and unlabeled cells both before and after cryopreservation (precryo n5, postthaw n2). (d) Table summarizing the viability and label uptake of groups of cells.

In consideration of early Phase 1 trials, the FDA states in their guidance for Cellular and Gene Therapy Products that evidence of the cell therapy products persistence and activity should be monitored. Further, the guidance document suggests monitoring the point of administration and site of intended activity, as well as other potential migratory sites or abnormal cell behavior [27]. Pharmacokinetic (PK) data for a cell therapy product in vivo would help answer some of these unknowns. The cell therapy results to date are promising [1-8]; however cellular therapies face a number of challenges before coming of a widely available treatment option. Manufacturing scale-up and obtaining pharmacokinetic data are two such challenges to be overcome, both obstacles considered throughout this manuscript.

Incorporation of an imaging agent into a clinical protocol requires that the imaging agent does not alter the cellular therapy, either in health or in function, and is capable of being imaged clinically [13]. Earlier experiments demonstrate 

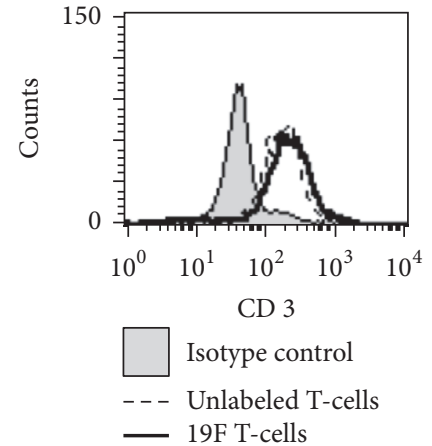

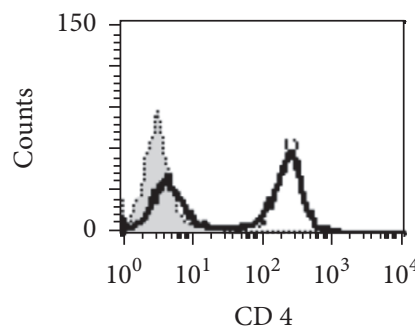

Isotype control

- - Unlabeled T-cells - 19F T-cells

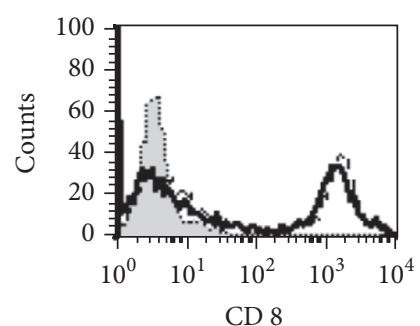

sotype control

- - Unlabeled T-cells - 19F T-cells
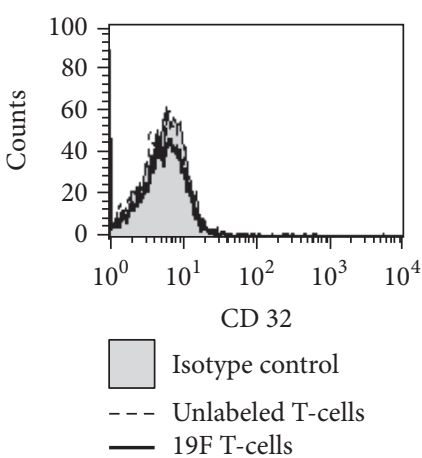

(a)

\begin{tabular}{lccccc}
\hline & & \multicolumn{4}{c}{ \% positive } \\
& & CD3 & CD4 & CD8 & CD32 \\
\hline \multirow{2}{*}{ Normal culture } & Control & $90.3 \pm 4.3$ & $34.1 \pm 4.2$ & $70.7 \pm 8.5$ & $7.0 \pm 2.3$ \\
& 19F & $86.6 \pm 8.0$ & $37.7 \pm 4.9$ & $69.3 \pm 8.3$ & $8.1 \pm 1.4$ \\
Cryopreserved and thawed & Control & $88.9 \pm 4.0$ & $36.1 \pm 1.9$ & $67.3 \pm 6.4$ & $6.7 \pm 2.7$ \\
& 19F & $84.2 \pm 7.9$ & $36.5 \pm 4.0$ & $66.5 \pm 7.5$ & $7.8 \pm 1.6$ \\
\hline
\end{tabular}

(b)

FIGURE 3: Phenotypic examination of labeled cells. Labeled and unlabeled T-cell populations are compared for the presence of the following phenotypic markers: CD3, CD4, CD8, and CD32. (a) Histograms comparing cellular populations labeled with conjugated antibodies for the depicted surface marker or isotype control. (b) Table summarizing the cellular populations (n2) presenting +/- CS-1000 ATM labeling and + /- cryopreservation.

the labels ability to maintain cellular health and function in multiple cell types $[18,20-24]$ and to be imaged at clinically relevant scan times [19]. For ease of use, having the label be easily incorporated into cellular processing was also important. CS-1000 ATM does not require the addition of transfection agents, which eases the incorporation process. Our studies demonstrate an additional simplification; by increasing the packaging and dose of deliverable reagent per vial, we were able to maintain the ease of use for large clinical scale manufacturing (Figure 1). A degree of the design of expansion protocols involve expansion of the culture in bioreactors in a clean room environment, factoring in the attachments (tubing) feeding into the bioreactors for the continued culture. Having a $25 \mathrm{~mL}$ dose of cellular label being administered to existing lines of a bioreactor via a vented spike eliminates the transfer of the culture to a biologic safety cabinet and lends itself to the industrial manufacturing process. Through the consideration of these scaling components, CS-1000 ATM was able to overcome previously undemonstrated questions of labeling cells at clinical scale.

The addition of the $19 \mathrm{~F}$ imaging agent to the manufacturing process did not alter common release criteria, namely, the cellular health or phenotype of the culture (Figures 2 and 3, resp.). An important aspect of adding an internalized label for cellular trafficking is not to alter these criteria. Cells were labeled with an average of $3.7 \times 10^{11} \mathrm{~F} / \mathrm{c}$. Cryopreservation of samples was also examined. When cells are being tested for release criteria, shipped, or stored for repeat dosing, cryopreservation may be necessary. Cellular phenotype, health, and cellular label were maintained upon thawing of cryopreserved samples (Figures 2 and 3). Other imaging modalities may lack the ability to be cryopreserved and require labeling of the cellular population after thawing due to the nature of the imaging agent (i.e., half-life) or concerns regarding the extent of cellular labeling [28].

Incorporating a cellular imaging agent to the therapeutic process allows $\mathrm{PK}$ data to be achieved through in vivo imaging of cellular therapies. Imaging enables the examination of a therapy's ability to reach its target (such as a solid tumor). Of equal importance, such methodology could account for potential off target delivery. In the selection of route of administration for cellular delivery, comparing various routes could help drive therapeutic outcome if one route demonstrates improved targeting. Furthermore, since therapeutic response may take 90 to 120 days, the possibility of using an imaging agent as a biomarker for efficacy when considering cancer treatment also holds great promise. Imaging also allows for the detection of cells failing to home/migrate; should a cellular therapy not reach the site of disease, readministration of the cellular therapy of alternative therapies could also be considered. Our goal by addressing the scalability of cellular labeling is that the technology can enable faster and more effective examination of cellular PK in man.

Imaging in clinical trials has the potential to strengthen safety and homing data. With one trial using 19F imaging completed [19] and another trial set to recruit (clinicaltrials.gov, NCT02035085) there are still steps being made in the implementation of this technology. Through the data developed in this manuscript, the concerns over clinical scale-up and incorporation of cellular labeling in clinical scale T-cell immunotherapy manufacturing have been addressed. 
As engineered T-cells are developed to target solid tumors, the ability to image with an agent that can transition from preclinical to clinical studies can help develop the pharmacokinetic data to necessary to accelerate the therapeutic path forward.

\author{
Abbreviations \\ CAR: Chimeric Antigen Receptor \\ TCR: T-Cell Receptor \\ ALL: $\quad$ Acute Lymphoblastic Leukemia \\ PK: $\quad$ Pharmacokinetic \\ 19F: $\quad$ Fluorine 19 \\ NK: Natural Killer \\ MRI: Magnetic Resonance Imaging \\ PET: $\quad$ Positron emission tomography \\ SPECT: Single photon emission computed tomography \\ CS: Cell Sense.
}

\section{Disclosure}

Human buffy coats were obtained from the Central Blood Bank (Pittsburgh, PA) for research purposes, and all applicable consent was collected by Central Blood Bank.

\section{Conflicts of Interest}

Charles F. O'Hanlon is an employee and shareholder of Celsense Inc. Brooke M. Helfer is an employee of Celsense Inc. Tamara Fedczyna, Shannon Eaker, and William D. Shingleton are employees of GE Healthcare. Cell Sense, the 19F MRI tracer agent described in the article, is manufactured by Celsense Inc. The Wave Bioreactor described in the article is manufactured by GE Healthcare. Celsense Inc. and GE Healthcare jointly provided fund and in-kind support for this research project.

\section{Authors' Contributions}

Charles F. O’Hanlon, Tamara Fedczyna, Shannon Eaker, William D. Shingleton, and Brooke M. Helfer participated in the composition, review, and editing of the manuscript. Brooke M. Helfer compiled the data and was responsible for the composition of the figures.

\section{Acknowledgments}

Funding for this work was provided by Celsense Inc. and a grant from GE Healthcare.

\section{References}

[1] S. A. Grupp, M. Kalos, D. Barrett et al., "Chimeric antigen receptor-modified $\mathrm{T}$ cells for acute lymphoid leukemia," The New England Journal of Medicine, vol. 368, no. 16, pp. 1509-1518, 2013.

[2] S. L. Maude, N. Frey, and P. A. Shaw, "Chimeric antigen receptor T cells for sustained remissions in leukemia," The New England Journal of Medicine, vol. 371, no. 16, pp. 1507-1517, 2014.
[3] A. L. Garfall, M. V. Maus, W.-T. Hwang et al., "Chimeric antigen receptor T cells against CD19 for multiple myeloma," The New England Journal of Medicine, vol. 373, no. 11, pp. 1040-1047, 2015.

[4] A. P. Rapoport, E. A. Stadtmauer, G. K. Binder-Scholl, O. Goloubeva, D. T. Vogl, and S. F. Lacey, "NY-ESO-1-specific TCRengineered $\mathrm{T}$ cells mediate sustained antigen-specific antitumor effects in myeloma," Nature Medicine, vol. 21, pp. 914-921, 2015.

[5] P. Kebriaei, H. Singh, M. H. Huls, M. J. Figliola, R. Bassett, S. Olivares et al., "Phase I trials using Sleeping Beauty to generate CD19-specific CAR T cells," The Journal of clinical investigation, vol. 126, no. 9, Article ID 5004935, pp. 3363-3376, 2016.

[6] X. Wang, L. L. Popplewell, J. R. Wagner et al., "Phase 1 studies of central memory-derived CD19 CAR T-cell therapy following autologous HSCT in patients with B-cell NHL," Blood, vol. 127, no. 24, pp. 2980-2990, 2016.

[7] J. N. Kochenderfer, M. E. Dudley, R. O. Carpenter et al., "Donorderived CD19-targeted $\mathrm{T}$ cells cause regression of malignancy persisting after allogeneic hematopoietic stem cell transplantation," Blood, vol. 122, no. 25, pp. 4129-4139, 2013.

[8] C. J. Turtle, L.-A. Hanafi, C. Berger et al., "CD19 CAR-T cells of defined CD4+:CD8+ composition in adult B cell ALL patients," The Journal of Clinical Investigation, vol. 126, no. 6, pp. 21232138, 2016.

[9] A. Bot, J. E. Brewer, Z. Eshhar et al., “Target discovery for T cell therapy: Next steps to advance Immunotherapies," Journal for Immuno Therapy of Cancer, vol. 3, no. 1, article no. 31, 2015.

[10] B. L. Levine, J. Miskin, K. Wonnacott, and C. Keir, "Global Manufacturing of CAR T Cell Therapy," Molecular Therapy Methods and Clinical Development, vol. 4, pp. 92-101, 2017.

[11] S. Eaker, E. Abraham, J. Allickson et al., "Bioreactors for cell therapies: Current status and future advances," Cytotherapy, vol. 19, no. 1, pp. 9-18, 2017.

[12] Society AC, "Facts and Figures 2016," Atlanta, American Cancer Society, 2016.

[13] J. V. Frangioni and R. J. Hajjar, "In vivo tracking of stem cells for clinical trials in cardiovascular disease," Circulation, vol. 110, no. 21, pp. 3378-3383, 2004.

[14] E. T. Ahrens and J. W. M. Bulte, "Tracking immune cells in vivo using magnetic resonance imaging," Nature Reviews Immunology, vol. 13, no. 10, pp. 755-763, 2013.

[15] A. V. Naumova, M. Modo, A. Moore, C. E. Murry, and J. A. Frank, "Clinical imaging in regenerative medicine," Nature Biotechnology, vol. 32, no. 8, pp. 804-818, 2014.

[16] M. Srinivas, A. Heerschap, E. T. Ahrens, C. G. Figdor, and I. J. M. de Vries, "19F MRI for quantitative in vivo cell tracking," Trends in Biotechnology, vol. 28, no. 7, pp. 363-370, 2010.

[17] C. M. Long and J. W. M. Bulte, "In vivo tracking of cellular therapeutics using magnetic resonance imaging," Expert Opinion on Biological Therapy, vol. 9, no. 3, pp. 293-306, 2009.

[18] E. T. Ahrens, R. Flores, H. Xu, and P. A. Morel, "In vivo imaging platform for tracking immunotherapeutic cells," Nature Biotechnology, vol. 23, no. 8, pp. 983-987, 2005.

[19] E. T. Ahrens, B. M. Helfer, C. F. O'Hanlon, and C. Schirda, "Clinical cell therapy imaging using a perfluorocarbon tracer and fluorine-19 MRI," Magnetic Resonance in Medicine, vol. 72, no. 6, pp. 1696-1701, 2014.

[20] P. Boehm-Sturm, L. Mengler, S. Wecker, M. Hoehn, and T. Kallur, "In vivo tracking of human neural stem cells with $19 \mathrm{~F}$ magnetic resonance imaging," PLoS ONE, vol. 6, no. 12, Article ID e29040, 2011. 
[21] M. N. Bouchlaka, K. D. Ludwig, J. W. Gordon et al., "19F-MRI for monitoring human NK cells in vivo," OncoImmunology, vol. 5, no. 5, Article ID el143996, 2016.

[22] B. M. Helfer, A. Balducci, A. D. Nelson et al., "Functional assessment of human dendritic cells labeled for in vivo $19 \mathrm{~F}$ magnetic resonance imaging cell tracking," Cytotherapy, vol. 12, no. 2, pp. 238-250, 2010.

[23] B. M. Helfer, A. Balducci, Z. Sadeghi et al., "19F MRI tracer preserves in vitro and in vivo properties of hematopoietic stem cells," Cell Transplantation, vol. 22, no. 1, pp. 87-97, 2013.

[24] M. Srinivas, P. A. Morel, L. A. Ernst, D. H. Laidlaw, and E. T. Ahrens, "Fluorine-19 MRI for visualization and quantification of cell migration in a diabetes model," Magnetic Resonance in Medicine, vol. 58, no. 4, pp. 725-734, 2007.

[25] P. Kaliński, C. M. U. Hilkens, A. Snijders, F. G. M. Snijdewint, and M. L. Kapsenberg, "IL-12-Deficient Dendritic Cells, Generated in the Presence of Prostaglandin E2, Promote Type 2 Cytokine Production in Maturing Human Naive T Helper Cells," The Journal of Immunology, vol. 159, no. 1, pp. 28-35, 1997.

[26] J. M. Janjic, M. Srinivas, D. K. K. Kadayakkara, and E. T. Ahrens, "Self-delivering nanoemulsions for dual fluorine-19 MRI and fluorescence detection," Journal of the American Chemical Society, vol. 130, no. 9, pp. 2832-2841, 2008.

[27] Guidance for Industry, "Preclinical Assessment of Investigational Cellular and Gene Therapy Products (DRAFT GUIDANCE)," in U.S. Department of Health and Human Services FDA, Center for Biologics Evaluation and Research, 2012.

[28] G. M. van Buul, G. Kotek, P. A. Wielopolski et al., "Clinically translatable cell tracking and quantification by MRI in cartilage repair using superparamagnetic iron oxides," PLoS ONE, vol. 6, no. 2, Article ID e17001, 2011. 


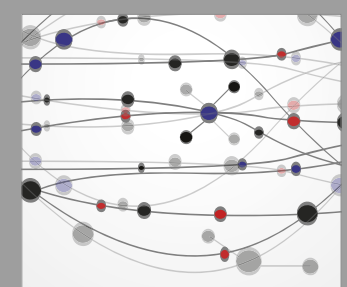

The Scientific World Journal
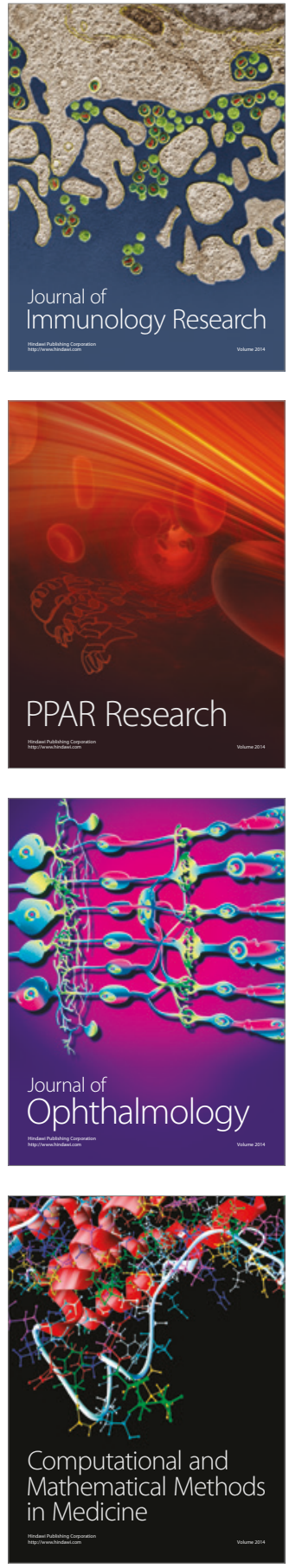

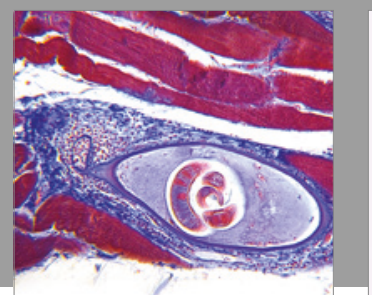

Gastroenterology Research and Practice
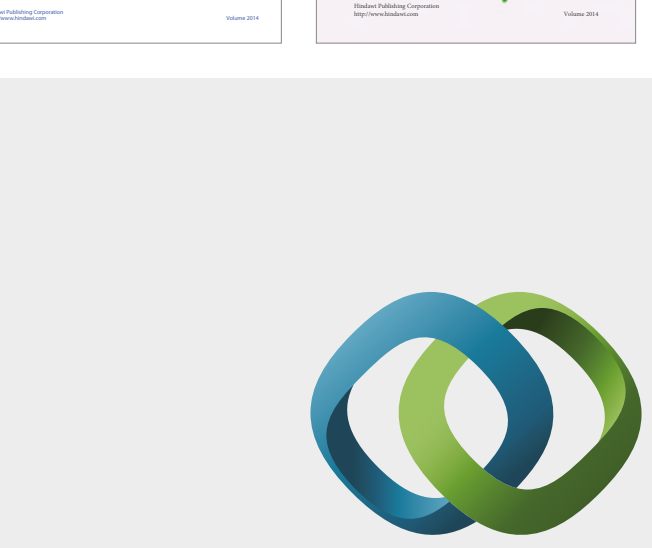

\section{Hindawi}

Submit your manuscripts at

https://www.hindawi.com
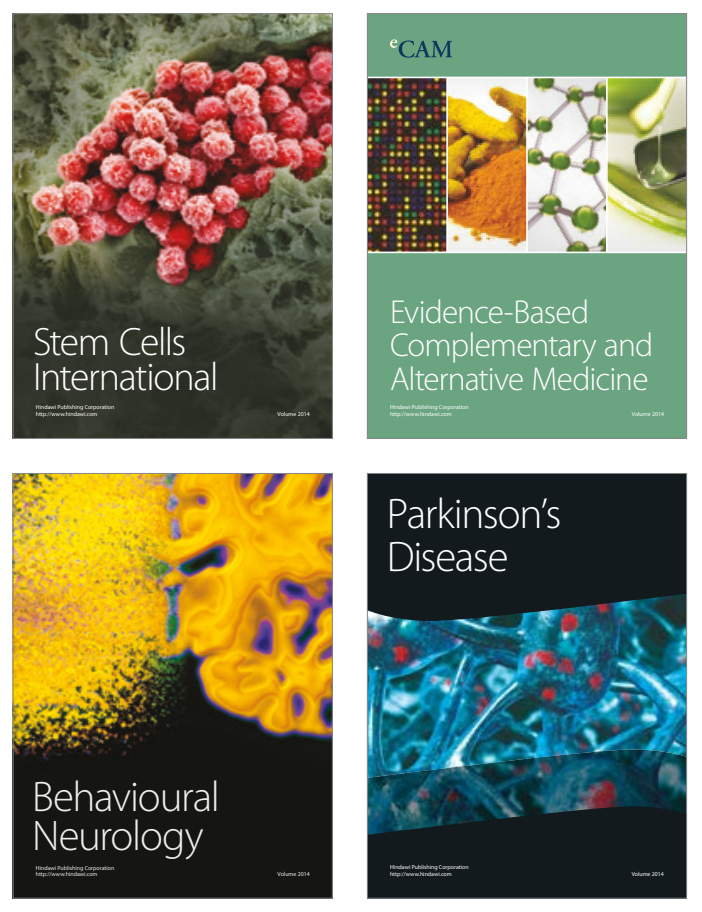
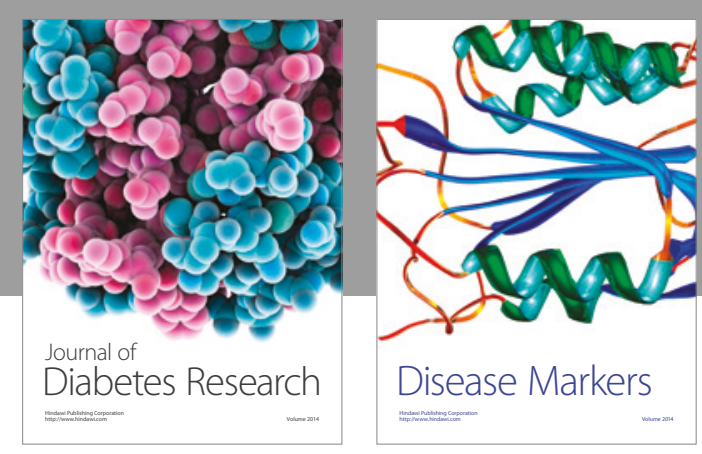

Disease Markers
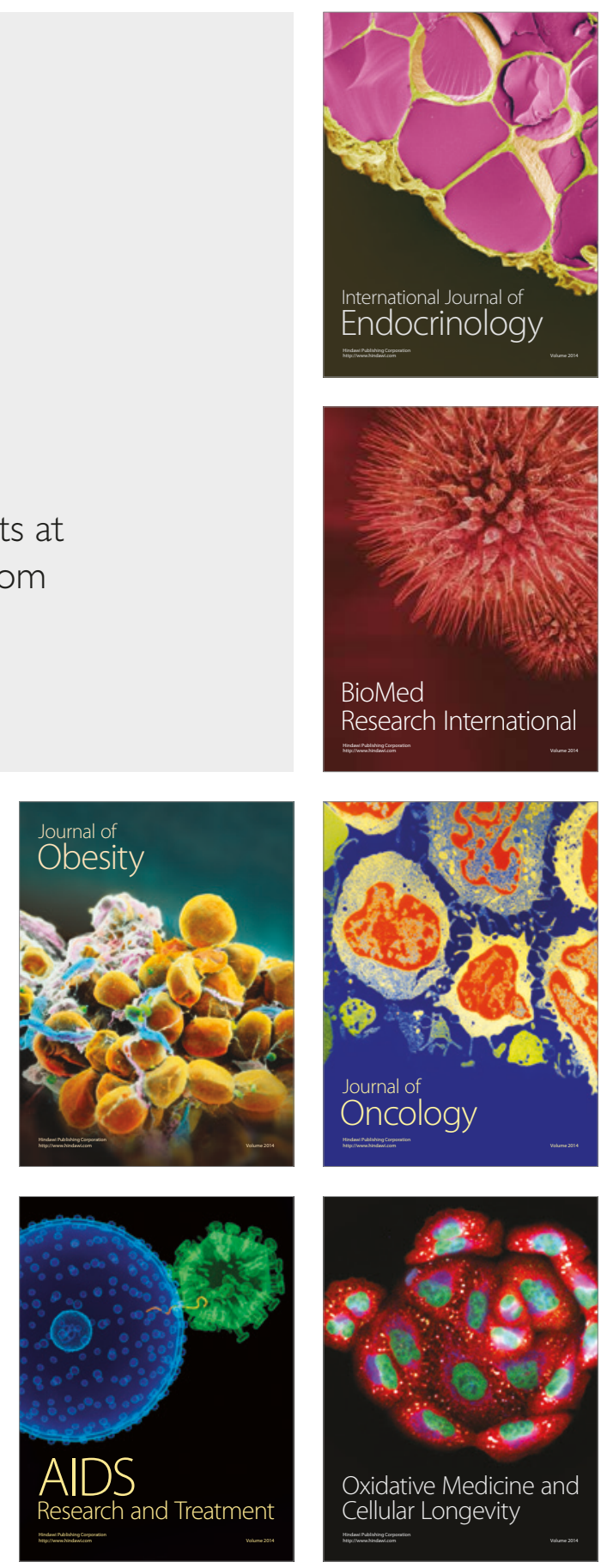